\title{
ACCESO A INTERNET Y LIBERTAD DE EXPRESIÓN EN LÍNEA: ¿DERECHOS HUMANOS?
}

\author{
María Emilia Miller Genta*
}

\begin{abstract}
RESUMEN. Las nuevas tecnologías, y en particular Internet, sirven a su propósito si nadie queda fuera de la sociedad de la información. De la misma manera, resulta crucial el respeto a la libertad de expresión en linea. El presente trabajo analiza distintos intentos de asegurar el acceso universal a Internet, con especial énfasis en el caso de Uruguay. Asimismo, explora el contenido de Internet en relación con el derecho a la libertad de expresión. Finalmente, recoge el debate respecto a si el acceso a Internet y la libertad de expresión en línea deben considerarse o no derechos humanos.
\end{abstract}

PALABRAS CLAVE. Acceso a Internet. Libertad de expresión en línea. Derechos Humanos. Nuevas tecnologías.

ABSTRACT. New technologies and the Internet in particular only commit to their purpose if everyone is part of the information society. Likewise, it is paramount to respect online freedom of expression. Throughout the following essay, attempts to ensure universal Internet access will be analyzed, with a special focus on the case of Uruguay. In addition, online content in relation to the right to freedom of expression will be explored. Finally, we will discuss if Internet access and online freedom of expression should or should not be considered human rights.

KEY WORDS. Internet access. Online freedom of expression. Human Rights. New technologies.

\section{INTRODUCCIÓN}

Resulta inequívoco que Internet plantea desafíos al Derecho en el siglo XXI: una anhela ser un ámbito no controlado, el otro busca controlar la sociedad. Las nuevas tecnologías y

* Traductora Pública en idioma ingles y estudiante de $6^{\circ}$ año de Abogacía en la Facultad de Derecho de la Universidad de la República. Adaptación de ensayo escrito durante International Summer School en University of East Anglia, julio de 2017. Correo electrónico: mariaemiliamillergenta@gmail.com 
el Derecho deben encontrar un equilibrio entre la libertad y el control, en especial porque el acceso universal a Internet repercute de manera importante en la libertad de expresión en línea y sus restricciones. En los últimos tiempos se ha desarrollado un debate en torno a la consideración como derechos humanos tanto del acceso a Internet como de la libertad de expresión en línea.

A lo largo de este trabajo, abordaremos la importancia del acceso universal a Internet y los esfuerzos tendientes a alcanzarlo mediante mecanismos jurídicos y políticas públicas. Luego, compararemos las limitaciones a la libertad de expresión en línea con las restricciones a la libertad de expresión tradicional. Finalmente, brindaremos un panorama de las posturas en cuanto al acceso a Internet y la libertad de expresión en línea como derechos humanos, tanto a nivel internacional como dentro del marco constitucional uruguayo.

\section{ACCESO A INTERNET}

El acceso a Internet forma parte de nuestra vida cotidiana y proporciona incontables oportunidades de información y comunicación. No obstante, su universalización requiere de los esfuerzos de los Estados y de la comunidad internacional. En este sentido, un informe de 2011 del Relator Especial sobre la promoción y protección del derecho a la libertad de opinión y de expresión en el Consejo de Derechos Humanos de la Asamblea General de Naciones Unidas indicó que "Internet solo puede responder a su finalidad si los Estados asumen su voluntad de elaborar políticas eficaces para obtener el acceso universal a Internet. A falta de políticas y planes de acción concretos, Internet pasará a ser un instrumento tecnológico al que solo podrá acceder una determinada élite, con lo cual se perpetuará la "brecha digital"."1

En efecto, varios países como Estonia, ${ }^{2}$ Francia, ${ }^{3}$ Grecia $^{4}$, Costa Rica, ${ }^{5}$ España ${ }^{6}$ y Finlandia ${ }^{7}$ han hecho referencia a la obligación de los Estados a proporcionar conexión a

1 Informe del Relator Especial sobre la promoción y protección del derecho a la libertad de opinión y de expresión, Frank La Rue, en el Consejo de Derechos Humanos de la Asamblea General de Naciones Unidas. Disponible en: http://www.acnur.org/t3/fileadmin/Documentos/BDL/2015/10048.pdf?view=1 Fecha de consulta: 19-DIC-2017

${ }^{2}$ Ley de Telecomunicaciones de Estonia de 2004 con sucesivas modificaciones. Disponible en: www.riigiteataja.ee/en/eli/501042015003/consolide Fecha de consulta 19-DIC-2017; ALEXANDER, Leigh \& SHORE, Matt. 'Internet access is now a basic human right' en The Guardian, 2016. Disponible en: www.theguardian.com/technology/audio/2016/aug/04/internet-access-human-right-2-tech-podcast Fecha de consulta: 16-JUL-2017

3 Sentencia del Consejo Constitucional de Francia de 2009. Disponible en: www.conseil-constitutionnel.fr/ conseilconstitutionnel/root/bank_mm/anglais/en2009_590dc.pdf Fecha de consulta: 19-DIC-2017; SPARKS, Ian. 'Internet access is a fundamental human right, rules French court' en The Daily Mail, 2009. Disponible en: www.dailymail.co.uk/news/article-1192359/Internet-access-fundamental-human-right-rules-French-court.html Fecha de consulta: 17-JUL-2017

4 Artículo 5.A.2 de la Constitución de Grecia de 2008. Disponible en: www.hellenicparliament.gr/User Files/f3c70a23-7696-49db-9148-f24dce6a27c8/001-156\%20aggliko.pdf Fecha de consulta: 19-DIC-2017

5 Sentencia de la Sala Constitucional de Costa Rica. "Retardo en la asignación de concesiones para bandas de frecuencia de telefonía celular. Internet como derecho fundamental." Disponible en: www.poderjudicial.go.cr/salaconstitucional/index.php/servicios-publicos/759-10-012790 Fecha de consulta: 13JUL-2017

6 Ley General de Telecomunicaciones de España de 2014. Disponible en: www.boe.es/buscar/ pdf/2014/ BOE-A-2014-4950-consolidado.pdf Fecha de consulta 19-DIC-2017 
Internet, ya sea en la legislación o en la jurisprudencia. Asimismo, el Tribunal de Apelaciones de Reino Unido determinó en Regina vs. Smith y otros (2011) que una prohibición total de Internet sería desproporcionada, debido a que es parte importante de la vida cotidiana. ${ }^{8}$

Siguiendo con esta línea de razonamiento, el caso de Uruguay resulta particularmente interesante, en tanto fue el escenario perfecto para la implementación del programa One Laptop Per Child ${ }^{9}$ de Nicholas Negroponte: una iniciativa sin fines de lucro que buscar brindar una computadora XO de bajo costo a cada niño en edad escolar con el objetivo de fomentar la alfabetización informática y el acceso a Internet en el mundo. Esto se convirtió en realidad en 2007, cuando el Estado uruguayo repartió las primeras computadoras en escuelas públicas, en virtud de lo que se conoce como Plan Ceibal. En 2009, Uruguay pasó a ser el primer país en el mundo en el que cada niño de las escuelas públicas tenía su propia computadora XO. Gracias al Plan Ceibal, el 100\% de las escuelas públicas uruguayas tiene conexión wifi, $93 \%$ de estas a través de fibra óptica. ${ }^{10}$

El plan fue objeto de críticas y es difícil de probar que haya significado mejoras educativas, pero sin dudas Ceibal potenció el acceso a internet entre los escolares uruguayos y sus familias. One Laptop Per Child en Uruguay fue un gran paso para promover la igualdad digital. A través de esta política, Uruguay logró un acceso prácticamente universal a internet, con conexión gratuita en todas las escuelas y la mayor parte de las plazas y edificios públicos.

\section{LIBERTAD DE EXPRESIÓN EN LÍNEA}

La libertad de expresión en línea es un factor fundamental de la sociedad de la información. De la misma forma que la libertad de expresión tradicional, la libertad de expresión en línea está sujeta a algunas limitaciones, pero existen diferentes puntos de vista acerca de si la libertad de expresión en línea debería estar restringida o no. Por un lado, la corriente ciberlibertaria ${ }^{11}$ entiende que no debe haber limitaciones porque la libertad de expresión debe ser aplicable en los nuevos medios de comunicación en las mismas formas en que se aplica a los medios tradicionales. Por otra parte, los Estados son conscientes de que Internet plantea nuevos riesgos y amenazas a los ciudadanos, y por lo tanto buscan

7 Ley de Mercados de la Comunicación de Finlandia de 2011. Disponible en: www.finlex.fi/en/laki/ kaannokset/2003/en20030393.pdf Fecha de consulta: 19-DIC-2017; AHMED, Saeed. 'Fast Internet access becomes a legal right in Finland' en $C N N$, 2009. Disponible en: www.edition.cnn.com/2009/ TECH/10/15/finland.internet.rights/index.html?_s=PM:TECH Fecha de consulta: 17-JUL-2017

8 Caso jurisprudencial Regina vs. Smith \& Ors de 19-JUL-2011. Disponible en: www.bailii.org/cgi-bin/ $\mathrm{m}$ a r k u p c g i ? d o c = / e w / c a s e s / E W C A / C r i m / $2011 /$ 1772.html\&query=2011+and+EWCA+and+Crim+and+1772\&method=boolean Fecha de consulta: 17-JUL-2017

9 One Laptop Per Child. Disponible en: www.one.laptop.org Fecha de consulta: 08-JUL-2017

10 Datos disponibles en el sitio web del Plan Ceibal: http://www.ceibal.edu.uy/es/articulo/ceibal-en-cifras Fecha de consulta: 08-JUL-2017

11 "Son ciberlibertarias aquellas personas que subscriben a una cierta ideología sobre las nuevas tecnologías, como por ejemplo Internet. Militan por el uso de la tecnología como medio de promover iniciativas personales o descentralizadas y menor dependencia de los gobiernos centrales." Definición tomada de Techopedia. Disponible en: https://www.techopedia.com/definition/29628/cyberlibertarian Fecha de consulta: 17-JUL-2017 
controlar el ciberespacio. ${ }^{12}$ Chris REED, jurista inglés especializado en Derecho Informático, plantea como posible solución considerar la normativa referente a la libertad de expresión en el Estado de cada usuario de Internet. Sin embargo, el propio autor reconoce que no sería viable de implementar y no evitaría que los usuarios contravengan disposiciones extranjeras. ${ }^{13}$

Varios Estados en el mundo han restringido el acceso a determinados sitios web, redes sociales y buscadores. Por ejemplo, los ciudadanos chinos no tienen acceso a Google, Facebook, Twitter, Instagram, Pinterest, Tumblr, YouTube, Blogspot y Wikipedia, entre otros. ${ }^{14}$ Las redes sociales como Facebook y Twitter también han estado prohibidas en países del Medio Oriente y el norte de África. ${ }^{15}$ Internet aloja contenidos potencialmente peligrosos, y el interminable intercambio de información puede resultar una amenaza para los sistemas no democráticos.

Aún no contamos con instrumentos internacionales relativos a los niveles mínimos de restricciones de la libertad de expresión en línea. No obstante, la normativa que regula la libertad de expresión en los medios de comunicación tradicionales habilita la posibilidad de incluir a las nuevas tecnologías. La libertad de expresión aparece regulada en el artículo 19 de la Declaración Universal de Derechos Humanos, el artículo 19 del Pacto Internacional de Derechos Civiles y Políticos, el artículo 13 (2) de la Convención Americana de Derechos Humanos, el artículo 10 (2) de la Convención Europea de Derechos Humanos, así como en otros instrumentos internacionales de derechos humanos. De conformidad con las normas referidas, las limitaciones al derecho a la libertad de expresión, y por lo tanto, a la libertad de expresión en Internet, solamente pueden disponerse por ley de manera de asegurar la certeza jurídica, los derechos de terceros, la seguridad, el orden público, la salud pública o la moral pública. Lo que es más, tales restricciones solo pueden tener lugar en circunstancias excepcionales y en relación con los principios de proporcionalidad y necesidad.

\section{4. ¿DERECHOS HUMANOS?}

Es posible esbozar varios argumentos a favor de considerar que el acceso a Internet y la libertad de expresión en línea cumplen con las expectativas de dignidad, libertad e igualdad que caracterizan a los derechos humanos en la actualidad. ${ }^{16}$ En primer lugar, partimos de la premisa de que tanto el Derecho como la conceptualización de los derechos humanos han evolucionado a través del tiempo, por lo que el ejercicio del derecho a la libertad de expresión debería poder extenderse a las nuevas tecnologías. Internet permite compartir y recibir información de manera instantánea y sin fronteras, así como promueve

12 EDWARDS, Lilian \& WAELDE, Charlotte. Law and the Internet, $3^{\text {rd }}$ ed, Hart Publishing, Oxford, 2009, p. 668

13 REED, Chris. Law in context: Internet Law, $2^{\text {nd }}$ ed, Cambridge University Press, Cambridge, 2004, pp. 256-268

14 MICHELINI, Michael, 'List of blocked websites in China' en Global from Asia, 2017. Disponible en: https://www.globalfromasia.com/list-blocked-sites-china Fecha de consulta: 19-JUL-2017

15 HELFT, Miguel, 'YouTube blocked in China, Google says' en New York Times, 2009. Disponible en: http://www.nytimes.com/2009/03/25/technology/internet/25youtube.html Fecha de consulta 17-JUL2017

16 PÉREZ LUÑO, Antonio, Derechos humanos, Estado de Derecho y Constitución, $9^{a}$ edición, Tecnos, Madrid, 2005, p. 50 
el acceso al conocimiento y la inclusión social, lo que la convierte en un ámbito propicio para la libertad de expresión. En este sentido, los seres humanos deberíamos tener en línea los mismos derechos que tenemos fuera de línea. Internet se convirtió en una parte crucial de la vida contemporánea y quizás sea hora de brindar protección más allá de la libertad de expresión y asegurar la conectividad. Desde este punto de vista, Internet parecería ser un requisito de libertad e igualdad en 2018.

No obstante, el principal argumento en contra de esta postura radica en que Internet no es un derecho humano en sí mismo, sino que es una vía para el goce de otros derechos, como por ejemplo el derecho a la libertad de expresión. En 2011, el Informe del Relator Especial sobre la promoción y protección del derecho a la libertad de opinión y de expresión, Frank LA RUE, en el Consejo de Derechos Humanos de la Asamblea General de Naciones Unidas, reconoce que Internet es una herramienta que posibilita que las personas ejerzan varios derechos humanos, entre ellos el derecho a la libertad de expresión. ${ }^{17}$

Un expositor de esta corriente es el científico en computación y pionero de Internet Vinton CERF, que considera que el acceso a Internet y la libertad de expresión en línea encajan mejor en la categoría de derechos civiles, esto es, aquellos que son conferidos por el Derecho en lugar de ser inherentes a la naturaleza humana. ${ }^{18}$ En efecto, la tecnología no es precisamente lo que consideraríamos esencial para la dignidad humana en un concepto tradicional. La conexión a Internet puede entenderse más como un lujo o un privilegio que un derecho, en virtud de que gran parte de la población mundial ni siquiera tiene acceso a necesidades humanas mucho más básicas. Otro asunto a destacar es el costo, puesto que la conexión a Internet y la banda ancha pueden ser inasequibles en algunos países.

En lo que respecta al Derecho positivo uruguayo, traemos a colación los dos importantes artículos que afilian nuestra Constitución al iusnaturalismo y que por tanto posibilitan la existencia de otros derechos y obligaciones incluso si no están reconocidos por escrito. Por un lado, el artículo 72 establece que la Constitución no excluye otros derechos que, aún si no están mencionados de forma expresa, sean inherentes a la personalidad humana o deriven de la forma republicana de gobierno. Por otra parte, el artículo 332 destaca que los derechos y las obligaciones no dejarán de existir por falta de reglamentación, situación ante la que regirán los fundamentos de las leyes análogas, los principios generales del Derecho y las doctrinas más recibidas. De considerar que el acceso a Internet es un derecho, estos artículos podrían contemplarlo. Asimismo, los artículos 28 y 29 de la Constitución de la República que abordan la correspondencia y la libertad de expresión hacen uso de expresiones amplias como "cualquier otra especie" y "cualquier otra forma de divulgación", a través de lo que es posible que la libertad de expresión en línea quede comprendida en la Constitución, pero en este caso Internet se consagra como un medio para ejercer derechos y no como un derecho en sí mismo.

17 Informe del Relator Especial sobre la promoción y protección del derecho a la libertad de opinión y de expresión, Frank La Rue, en el Consejo de Derechos Humanos de la Asamblea General de Naciones Unidas. Disponible en: http://www.acnur.org/t3/fileadmin/Documentos/BDL/2015/10048.pdf?view=1 Fecha de consulta: 19-DIC-2017

18 CERF, Vinton, 'Internet access is not a human right' en New York Times, 2012. Disponible en: http:/ /www.nytimes.com/2012/01/05/opinion/internet-access-is-not-a-human-right.html Fecha de consulta: 12-JUL-2017 


\section{CONCLUSIONES}

Los Estados deben asegurar el acceso universal a Internet de manera de promover la inclusión digital y social. El caso de Uruguay refleja cómo el Plan Ceibal otorgó una computadora a cada niño de las escuelas públicas y logró brindar conexión a Internet gratuita en todas las escuelas, lo que a su vez incitó la libertad de expresión en línea. Los contenidos de Internet están sujetos a limitaciones, pero estas tienen que cumplir con las disposiciones expuestas en los instrumentos internacionales relativos a las restricciones a la libertad de expresión.

El debate acerca de si el acceso a Internet y la libertad de expresión en línea son derechos humanos todavía se encuentra abierto a discusión. Internet facilita el ejercicio de otros derechos, como el derecho a la libertad de expresión, que además puede extenderse a la libertad de expresión en línea. Sin perjuicio de no estar previstos en forma expresa, el acceso a Internet y la libertad de expresión en línea pueden ingresar al marco constitucional uruguayo de conformidad con los artículos 28, 29, 72 y 332 de nuestra Constitución. En suma, el acceso a Internet y la libertad de expresión en línea no están reconocidos como derechos humanos, pero sin duda alguna permiten la realización de varios derechos en nuestra sociedad de comienzos del siglo XXI.

\section{BIBLIOGRAFÍA CONSULTADA}

AHMED, Saeed. 'Fast Internet access becomes a legal right in Finland' en $C N N, 2009$. Disponible en: www.edition.cnn.com/2009/TECH/10/15/finland.internet.rights/index (Fecha de consulta: julio 2017)

ALEXANDER, Leigh \& SHORE, Matt. 'Internet access is now a basic human right' en The Guardian, 2016. Disponible en:www.theguardian.com/technology/audio/2016/aug/ 04/internet-access-human-right-2-tech-podcast (Fecha de consulta: julio 2017).

BEST, Michael. 'Can the Internet be a human right?' en Semantic Scholar. Disponible en: www.pdfs.semanticscholar.org/93b3/4da632398bd954de0811214994a8269266b3 (Fecha de consulta: julio 2017).

BORG PSAILA, Stephanie. 'Right to access the Internet: the countries and laws that proclaim it' en Diplomacy, 2011. Disponible en: https://www.diplomacy.edu/blog/right-access-internetcountries-and-laws-proclaim-it (Fecha de consulta: julio 2017).

BAINBRIDGE, David. Introduction to Information Technology Law, $6^{\text {th }}$ ed, Pearson, London, 2008

BARENDT, Eric. Freedom of Speech, Oxford University Press, Oxford, 1985

CERF, Vinton, 'Internet access is not a human right' en New York Times, 2012. Disponible en: www.nytimes.com/2012/01/05/opinion/internet-access-is-not-a-human-right (Fecha de consulta: julio 2017).

DE HERT, Paul \& KLOZA, Dariusz, 'Internet (access) as a new fundamental right. Inflating the current rights framework?' en European Journal of Law and Technology, Vol. 3. No. 3, 2012. Disponible en: www.ejlt.org/article/view/123/268 (Fecha de consulta: julio 2017).

EDWARDS, Lilian \& WAELDE, Charlotte. Law and the Internet, $3^{\text {rd }}$ ed, Hart Publishing, Oxford, 2009, p. 668

HELFT, Miguel. 'YouTube blocked in China, Google says' en New York Times, 2009. Disponible en: www.nytimes.com/2009/03/25/technology/internet/25youtube.html (Fecha de consulta: julio 2017).

Informe del Relator Especial sobre la promoción y protección del derecho a la libertad de opinión y de expresión, Frank La Rue, en el Consejo de Derechos Humanos de la Asamblea General 
de Naciones Unidas, 2011. Disponible en: http://www.acnur.org/t3/fileadmin/Documentos/ BDL/2015/10048.pdf?view=1 (Fecha de consulta: julio 2017).

JOYCE, Daniel. 'Internet freedom and human rights', en European Journal of International Law, 2015. Disponible en: www.academic.oup.com/ejil/article/26/2/493/423010/Internet-Freedomand-Human-Rights (Fecha de consulta: julio 2017).

KAHN, Richard \& KELLNER, Douglas. 'New Media and Internet activism: from the "Battle of Seattle” to blogging', 2004. Disponible en: www.journals.sagepub.com /doi/pdf/10.1177/ 1461444804039908 (Fecha de consulta: julio 2017).

KEVAN, Tim \& McGRATH, Paul. Email, the Internet and the Law, Emis Professional Publishing, Hertfordshire, 2001

NICOL, Andrew, MILLAR, Gavin \& SHARLAND, Andrew. Media Law and Human Rights, $2^{\text {nd }}$ edn, Oxford 2009

PÉREZ LUÑO, Antonio. Derechos humanos, Estado de Derecho y Constitución, $9^{a}$ edición, Tecnos, Madrid, 2005, p. 50

REED, Chris. Law in context: Internet Law, $2^{\text {nd }}$ ed, Cambridge University Press, Cambridge, 2004, pp. 256-268

MICHELINI, Michael. 'List of blocked websites in China' en Global from Asia, 2017. Disponible en: https://www.globalfromasia.com/list-blocked-sites-china (Fecha de consulta: julio 2017).

ROCHE, Maria. 'Full Internet ban for sex offenders ruled unlawful', 2013. Disponible en: www.ukhumanrightsblog.com/2011/08/12/full-internet-ban-for-sex-offenders-ruled-unlawful (Fecha de consulta: julio 2017).

ROTHKOPF, David. 'Is unrestricted Internet access a modern human right?, 2015. Disponible en: www.foreignpolicy.com/2015/02/02/unrestricted-internet-access-human-rights-technologyconstitution (Fecha de consulta: julio 2017).

SPARKS, Ian. 'Internet access is a fundamental human right, rules French court' en The Daily Mail, 2009. Disponible en: www.dailymail.co.uk/news/article-1192359/Internet-access-fundamental-human-right-rules-French-court.html (Fecha de consulta: julio 2017).

WARMAN, Matt. 'Tim Berners-Lee defends net neutrality' en The Telegraph, 2010. Disponible en: www.telegraph.co.uk/technology/internet/8003908/Tim-Berners-Lee-defends-netneutrality.html (Fecha de consulta: julio 2017).

Fecha de recepción: 9 febrero 2018

Fecha de aceptación: 23 marzo 2018. 
